\title{
Presentación del proyecto de investigación del grupo TesUN (Universidad de Navarra)
}

\author{
Maite Iraceburu Jiménez, Concepción Martínez Pasamar, Cristina \\ Tabernero Sala \\ Università degli Studi di Firenze, Universidad de Navarra
}

This paper aims at presenting the Project of Corpus Linguistics in Spanish developed by the research group TesUN of the Universidad de Navarra, which also is a part of the Red CHARTA. The corpus consists of personal ancient documents which date back to $17^{\text {th }}, 18^{\text {th }}$ and $19^{\text {th }}$ centuries. These texts were preserved in private archives at private homes in Navarra (Spain). The established corpus is characterized by the heterogeneity of its textual typology, which ranges from notarial documents -copies, testaments, marriage contractsto private writing -letters, receipts, bills or obituaries-, or, in the case of the former parsonages, sermons and biblical exegesis, as well as letters. Therefore, this corpus opens a wide range of study to paleographers, historians -in particular to those interested in the history of private life and the history of mentalities-, linguists or ethnographers and ethnologists.

Keywords: Corpus linguistics, History of the language, personal documentation, Navarra, Red CHARTA.

\section{Introducción}

El objetivo del presente artículo es dar a conocer el proyecto de Lingüística de corpus en español desarrollado por el grupo TesUN de la Universidad de Navarra, perteneciente a la Red CHARTA, que se propone

[1]a publicación en red de un corpus de textos y documentos antiguos de los siglos XII al XIX de España e Hispanoamérica, con un sistema de presentación triple riguroso (paleográfica, crítica y facsimilar) con el fin de satisfacer distintas necesidades de investigadores y usuarios en general ${ }^{1}$.

\footnotetext{
${ }^{1}$ En <https://www.redcharta.es/que-es-la-red-charta-y-cuales-son-sus-objetivos/> [08/06/18].
} 
A lo largo de las páginas de este artículo se expondrá el origen del grupo TesUN dentro de la Red CHARTA, así como los documentos que conforman el objeto de su estudio, la orientación de las publicaciones a las que está dando lugar, y, por último, las nuevas perspectivas de investigación.

\section{Origen de TesUN dentro de la Red CHARTA. Objetivos}

En primer lugar, resulta necesario presentar la Red CHARTA, definida como:

[u]n proyecto global de edición de textos archivísticos hispánicos que pretende integrar una sólida fundamentación filológica y los desarrollos informáticos necesarios para proporcionar así a los investigadores e interesados por la lengua, la historia y la cultura unos textos digitales fiables, comprobables y que se pueda citar directamente en estudios de diferente ámbito ${ }^{2}$.

Por lo que respecta al origen de dicho proyecto de edición de documentos, este nació en julio de 2005 en la Universidad de Deusto (País Vasco) a raíz de la reflexión sobre los usos filológicos con respecto a la marcación de textos para su edición digital. En mayo de 2006 la Universidad de Alcalá acogió el segundo encuentro de este grupo de historiadores de la lengua, en el que se fijaron los criterios de edición de las fuentes documentales seleccionadas, que abarcan documentos públicos y privados de todas las épocas del español hasta el siglo XX, ordenados de acuerdo con la siguiente tipología: textos legislativos, cartas de compraventa y contratos, actas y declaraciones, cartas privadas, testamentos e inventarios, informes y relaciones, estatutos, certificaciones notas y breves y, por último, un grupo indeterminado de "otros"; estas habrían de ofrecer una triple presentación: (a) facsímil, (b) transcripción paleográfica y (c) presentación crítica. Finalmente, en diciembre de 2006 en Murcia y en mayo de 2007 en Valladolid se establecieron los criterios de edición definitivos, tal como figuran en la dirección electrónica correspondiente ${ }^{4}$.

Desde entonces, los diferentes grupos que forman la Red CHARTA ${ }^{5}$ han contribuido a la elaboración de un corpus documental disponible en forma

\footnotetext{
${ }^{2}$ En <https://www.redcharta.es/que-es-la-red-charta-y-cuales-son-sus-objetivos/> [08/06/18].

${ }^{3}$ Información disponible en <https://bit.ly/2PPh5fp> [29/08/18].

${ }^{4}$ https://bit.ly/2ynRikq [29/08/18].

${ }^{5}$ CSIC, Instituto Cervantes de Manila, King's College London, Universidad Complutense de Madrid, Universidad de Alcalá, Universidad de Antioquia, Universidad Autónoma de Barcelona, Universidad de Deusto, Universidad de Gotemburgo, Instituto Caro y Cuervo, Universidad de las Islas Baleares, Universidad de Lausana, Universidad de Las Palmas de Gran Canaria, Universidad de Los Andes, Universidad de Navarra, Universidad de Neuchâtel,
} 
electrónica ${ }^{6}$. En la actualidad este cuenta con 1346094 formas en 2076 documentos transcritos ${ }^{7}$, comprendidos entre los años 822 y 1871 , que proceden no solo de los diferentes puntos de la Península ibérica, sino también de las islas Canarias, de Hispanoamérica ${ }^{8}$ e incluso de Portugal e Inglaterra ${ }^{9}$.

A su vez, la Red CHARTA celebra reuniones y congresos bianuales en los que se exponen los resultados de investigación de los documentos transcritos. El primer congreso de CHARTA, Tradición e innovación: nuevas perspectivas en el estudio y edición de documentos antiguos, tuvo lugar en el Consejo Superior de Investigaciones Científicas de Madrid, en noviembre de 2009. A este le siguieron el congreso de Neuchâtel (Suiza) de septiembre de 2011, el de Salamanca de junio de 2013, el de Gotemburgo (Suecia) de octubre de 2015 y, por último, el de Lausana (Suiza), de junio de 2017, bajo el título Reescribiendo la historia de la lengua a partir de la edición de documentos. El próximo congreso tendrá lugar en Sevilla en 2019.

En todos estos encuentros se ha puesto en común el producto de la investigación de cada uno de los diferentes grupos, que trabajan para la consecución de los objetivos comunes de la Red CHARTA, que son los siguientes ${ }^{10}$ :

a. Establecer una metodología común para la edición de textos y la creación de corpus documentales. Triple presentación del documento: transcripción paleográfica, presentación crítica y reproducción facsimilar.

b. Fijar un estándar de presentación gráfica para la transcripción paleográfica y la edición crítica, con criterios filológico-lingüísticos rigurosos y homogéneos.

Universidad de Padua, Universidad Autónoma de Querétaro, Universidad de Salamanca, Universidad de Santiago de Compostela, Universidad de Sevilla, Universidad de Granada, Universidad de Málaga, Universidad de Tokio, Universidad Nacional Autónoma de México. La Red está abierta a nuevos grupos que soliciten la incorporación.

${ }^{6}$ Cfr. <http://www.corpuscharta.es/>

${ }^{7}$ Para más detalle sobre grupos, subgrupos y número de formas, cfr. el apartado de estadísticas. [Última fecha de consulta: 31/08/18].

La Red CHARTA organiza reuniones científicas en forma de encuentros, bianuales y de carácter interno, y de congresos internacionales abiertos a la comunidad científica en general, celebrados también cada dos años, alternantes, por tanto, con los encuentros. Ver la historia del proyecto CHARTA en <https://bit.ly/2BX72lh> [consulta 15/08/2018].

${ }^{8}$ Colombia, Cuba, Ecuador, El Salvador, Guatemala, México, Panamá, República Dominicana y Venezuela.

${ }^{9} \mathrm{Cfr} .<\mathrm{http}: / /$ www.corpuscharta.es/consultas.html>

${ }^{10} \mathrm{Cfr}$. <https://www.redcharta.es/que-es-la-red-charta-y-cuales-son-sus-objetivos/> 
c. Elaborar un corpus creciente en Internet de fuentes documentales que abarque la variedad geográfica del español, tanto de España como de América, en un marco cronológico amplio, con herramientas informáticas comunes para el análisis lingüístico de los textos.

d. Desarrollar estudios de paleografía, diplomática, grafemática, fonología y fonética, morfología, sintaxis, léxico y semántica, tradiciones discursivas, pragmática y sociolingüística históricas sobre los materiales del corpus.

Precisamente a la consecución de dichos propósitos contribuye el grupo TesUN de la Universidad de Navarra. Este nació en el año $2012^{11}$ y desde entonces se ha ocupado de la transcripción y edición de un archivo documental privado (CORAPRINA) perteneciente a la zona nororiental de Navarra. El trabajo sobre estos textos, según las normas de CHARTA, se publica en la página web del grupo investigador (https://bit.ly/2MEX398); hasta el momento se han publicado quince documentos notariales -cinco cartas de pago, tres certificados y permisos, un contrato, una deuda, una dote, un pleito, dos sentencias, un testamento-, fechadas entre 1550 y 1840 , y cien cartas personales del siglo XIX.

\section{Documentos del grupo TesUN. Establecimiento del corpus: dificultades y retos}

El grupo TesUN pudo dar inicio a su andadura gracias al encuentro de la documentación del Archivo de Casa Lujena, en la localidad navarra de Redín, donde se encontró una ingente documentación privada que abarcaba un arco temporal comprendido entre los siglos XVII y XIX. Todos estos documentos procedían de archivos conservados en casas particulares de Navarra. De esta manera, el corpus establecido se caracteriza por la heterogeneidad de su tipología textual, que abarca desde documentos notariales, como traslados, testamentos o contratos matrimoniales, hasta escritos de carácter privado, como cartas, recibos, billetes o esquelas; además, por tratarse de una antigua casa parroquial -la casa del $a b a d^{12}-$, sermones y exégesis bíblicas.

\footnotetext{
${ }^{11}$ De la mano de Cristina Tabernero Sala, a quien se sumaron Concepción Martínez Pasamar y Carmen Llamas Saíz, todas ellas profesoras de la Universidad de Navarra. Con el tiempo el grupo se amplió con la incorporación de Naiara Ardanaz, historiadora, que se encarga de la transcripción de los documentos en euskera. A su vez, el grupo ha contado con la colaboración de estudiantes de doctorado (Maite Iraceburu Jiménez) y de alumnos colaboradores del Departamento de Filología de la Universidad de Navarra (Steven García Chalarca y Johanna Pérez Garciarena).

${ }^{12}$ En Navarra y Aragón, se denominaba de este modo al párroco.
} 
La mayoría de los documentos están redactados en español, si bien es cierto que se han compendiado textos en euskera, ya que estos proceden de la parte de Navarra en la que ambas lenguas estaban en contacto, aspecto que otorga a estos escritos un especial interés sociolinguiístico y dialectológico. Es este aspecto uno de los retos a los que se enfrenta este grupo de investigación, ya que el vascuence que se ofrece en estos textos no se corresponde con el "euskara batua"13, la variedad estándar de la lengua vasca fijada en el siglo XX, sino que se trata de una variante del vascuence hablado en la zona de Redín, en la zona prepirenaica del noreste navarro y extinguida en la actualidad.

De este modo, de acuerdo con los criterios de la Red CHARTA, los documentos que configuran el corpus de TesUN se presentan en una triple versión: una transcripción paleográfica, en primer lugar; una presentación crítica en la que se edita el texto y, finalmente, se muestra el facsímil que permite el acceso directo a los textos.

\section{Resultados de la actividad del grupo TesUN}

A su vez, el análisis (socio)lingüístico y discursivo de las fuentes ha da lugar a la publicación de artículos sobre diferentes aspectos como las metáforas de los sermones (Iraceburu 2016), la historia de la ortografía (Martínez Pasamar y Tabernero 2013), las lenguas de Navarra (Martínez Pasamar y Tabernero 2012), la oralidad y dialoguicidad fingida (Martínez Pasamar, en prensa), el discurso testamentario (Tabernero 2016), la variación léxica (Tabernero 2012), el léxico espacial (Tabernero 2012), la tipología textual (Tabernero 2009) o los procedimientos de atenuación (Tabernero, en prensa) e intensificación (Martínez Pasamar, en prensa) en correspondencia privada.

\section{Apuntes finales. Perspectivas del grupo TesUN}

El objetivo principal en los próximos años es el de llevar a cabo una labor pormenorizada de catalogación y edición del corpus establecido, de modo que este pueda ampliarse mediante la búsqueda de documentos de su misma naturaleza en otros archivos privados de Navarra, en los que se tratará de encontrar "mano femenina" (particularmente correspondencia). Es por ello por lo que la actividad de TesUN se incluye dentro del proyecto de investigación "Universos

\footnotetext{
13 "Euskera unificado" o "unido" en lengua vasca. Se correspondería con un vasco estándar, una lengua koiné basada en los dialectos centrales del euskera (el dialecto navarro, el navarrolabortano y el dialecto central del euskera). Este estaría influido a su vez por el labortano clásico del siglo XVII.
} 
discursivos e identidad femenina: élites y cultura popular (1600-1850)", para el que se transcribirán y estudiarán aquellos textos (cantares, libelos, injurias, correspondencia) redactados por mujeres entre los siglos XVII y XIX, con el fin de estudiar el lenguaje femenino de las élites rurales desde una perspectiva lingüística, histórica y social.

Por otro lado, desde la perspectiva sociolingüística y sociopragmática, otro de los objetivos es abordar la imagen de la mujer transmitida en la documentación tomando como base del análisis tanto las referencias textuales al género femenino como los documentos -casi con exclusividad cartas, y estas también escasasredactadas por mujeres. El estudio de las referencias en el texto combinará la descripción lingüística con las condiciones de enunciación, con el contexto social $\mathrm{y}$, hasta donde sea posible, con el individual. Resulta evidente que aspectos como el léxico resultan esenciales en el dibujo colectivo de la mujer, resultado del papel social que se le ha atribuido en cada época.

Por su parte, de modo complementario al anterior, la caracterización discursiva de la mano femenina, siempre en relación con elementos extralingüísticos, contribuirá a determinar la identidad lingüística de género.

En definitiva, de acuerdo con la exposición realizada, el corpus del grupo TesUN abre un amplio abanico de estudio a paleógrafos, historiadores (especialmente a aquellos interesados en la historia de la vida privada y de las mentalidades), lingüistas, etnógrafos, etnólogos y, en definitiva, a todos aquellos que deseen conocer de primera mano la lengua y las costumbres locales y ponerlas en relación con las de otras áreas.

\section{Referencias}

Iraceburu Jiménez, M. 2016. Metáforas y contexto social en sermones del siglo XVIII, Príncipe de Viana. 265, 733-756.

Martínez Pasamar, C. y C. Tabernero 2013. Ortografía y alfabetización a partir de la edición de fuentes escritas del siglo XIX. Cilengua (Cuadernos del Instituto de Historia de la Lengua), 6-8, 199-227.

Martínez Pasamar, C. y C. Tabernero 2012. Hablar en Navarra: las lenguas de un reino (12121512). Pamplona, Gobierno de Navarra.

Martínez Pasamar, C. (en prensa): Ea, pues, alerta. Manifestaciones de oralidad y dialoguicidad fingida en un sermón de entresiglos (XVIII-XIX). Nuevos comentarios de textos hispánicos. Homenaje a Miguel Ángel Garrido.

Tabernero, C. 2016. El testamento como género discursivo en documentación peninsular (de la Edad Media al siglo XVIII). Onomazein, 34, 70-85.

Tabernero, C. 2013. Documentos navarros. Cartas privadas. En Isasi, C. y J. L. Ramírez Luengo. Una muestra documental del castellano norteño en el siglo XIX. Lugo: Axac, 177211. 
Tabernero, C. 2012. Documentación jurídica y variación léxica en los siglos XVI y XVII. En Martínez Pasamar, C. y C. Tabernero (eds), Por seso e por maestría. Homenaje a la profesora Carmen Saralegui. Pamplona: EUNSA, 2012, 523-554.

Tabernero, C. 2012 La concepción del espacio a través del léxico y la configuración geolectal, en Gómez Seibane, S. y C. Sinner: Estudios sobre tiempo y espacio en el español norteño. San Millán de la Cogolla: Cilengua, 69-90.

Tabernero, C. 2012. Tradición y actualidad en los estudios lingüísticos sobre Navarra. Archivo de Filología Aragonesa, 68, 185-212.

Tabernero, C. 2009. Tradiciones discursivas y tipos de texto en un documento jurídico de 1578. En Arellano, I., García Ruiz, V. y C. Saralegui (eds), Ars bene docendi. Homenaje al profesor Kurt Spang. Pamplona: EUNSA, 553-556.

Tabernero, C. 2008. Disponibilidad y contacto de lenguas. Oihenart. Cuadernos de Lengua y Literatura, 23, 245-285.

Tabernero, C. (en prensa). Fórmulas de cortesía y mecanismos de atenuación en la retórica epistolar del siglo XIX.

\section{Consultas en la web}

Grupo TesUN (Universidad de Navarra): <http://www.unav.edu/web/facultad-de-filosofia-yletras/documentos-privados-antiguos/presentacion> [última consulta: 27/04/18].

Red CHARTA: <https://www.redcharta.es/> [última consulta: 27/04/18]. 